\title{
Pacific
}

Journal of

Mathematics

\section{FIXED POINTS OF BOUNDARY-PRESERVING MAPS OF SURFACES}

ROBERT F. BROWN AND BRIAN SANDERSON 


\title{
FIXED POINTS OF BOUNDARY-PRESERVING MAPS OF SURFACES
}

\author{
ROBERT F. BROWN AND BRIAN J. SANDERSON
}

Let $X$ be a compact 2 -manifold with nonempty boundary $\partial X$. Given a boundary-preserving map $f:(X, \partial X) \rightarrow(X, \partial X)$, let $M F_{\partial}[f]$ denote the minimum number of fixed points of all boundarypreserving maps homotopic to $f$ as maps of pairs and let $N_{\partial}(f)$ be the relative Nielsen number of $f$ in the sense of Schirmer [S]. Call $X$ boundary-Wecken, $\mathbf{b W}$, if $M F_{\partial}[f]=N_{\partial}(f)$ for all boundarypreserving maps of $X$, almost $b \boldsymbol{W}$ if $M F_{\partial}[f]-N_{\partial}(f)$ is bounded for all such $f$, and totally non-bW otherwise. We show that if the euler characteristic of $X$ is non-negative, then $X$ is bW. On the other hand, except for a relatively small number of cases, we demonstrate that the 2-manifolds of negative euler characteristic are totally non-bW. For one of the remaining cases, the pants surface $P$, we use techniques of transversality theory to examine the fixed point behavior of boundary-preserving maps of $P$, and show that $P$ is almost bW.

1. Introduction. Throughout this paper, we will be working in the setting of compact manifolds. Given a map $f: X \rightarrow X$ of a compact manifold $X$, we denote the Nielsen number of $f$ by $N(f)$ and let $M F[f]$ be the minimum number of fixed points of all maps homotopic to $f$. The manifold $X$ is said to be Wecken if $M F[f]=N(f)$ for all maps $f: X \rightarrow X$. Wecken [W] proved that all $n$-manifolds are Wecken for $n \geq 3$ and Jiang [J] proved that a 2-manifold is Wecken if and only if its euler characteristic is non-negative. The interval is obviously Wecken and it is a classical result that the circle is Wecken.

Now suppose that the manifold $X$ has nonempty boundary $\partial X$ and that $f$ is boundary-preserving, that is, $f$ maps $\partial X$ to itself so $f$ is a map of pairs $f:(X, \partial X) \rightarrow(X, \partial X)$. We denote the relative Nielsen number by $N_{\partial}(f)$ and write $M F_{\partial}[f]$ for the minimum number of fixed points of all maps homotopic to $f$ as maps of pairs. We say that a manifold $X$ with nonempty boundary is boundary-Wecken, abbreviated $\mathrm{bW}$, if $M F_{\partial}[f]=N_{\partial}(f)$ for all maps $f:(X, \partial X) \rightarrow(X, \partial X)$. It is obvious that the interval is bW and Schirmer [S] proved that all $n$-manifolds are bW for $n \geq 4$. The purpose of this paper is to investigate the $b W$ property for boundarypreserving maps of 2-manifolds. We begin, however, with a remark 
about 3-manifolds. Although all 3-manifolds are Wecken, it is easy to see that not all of them are bW. To construct a simple example, let $X$ be a closed 2-manifold of negative euler characteristic. Let $g: X \rightarrow X$ be a map, as in $[\mathbf{J}]$, with $M F[g]>N(g)$ and define $f: X \times I \rightarrow X \times I$ by $f(x, t)=\left(g(x), t^{2}\right)$ for $x \in X$ and $t \in I$. Then $N_{\partial}(f)=2 N(g)$ since $f$ has no fixed points on the interior of $X \times I$. On the other hand, a boundary-preserving map of $X \times I$ must take each boundary component to itself, so $M F_{\partial}[f]=2 M F[g]>N_{\partial}(f)$. Thus the properties Wecken and bW are not equivalent in the setting of 3-manifolds with boundary.

In contrast to 3-manifolds, it is considerably more difficult to find 2manifolds with boundary which are not either both Wecken and bW or neither Wecken nor bW. In $\S 2$, we will prove that the 2-manifolds with boundary that have non-negative euler characteristic: the disc, annulus and Möbius band, are bW as well as Wecken. In $\S 3$, we show that for many surfaces with negative euler chararcteristic, the coincidence of the Wecken and bW properties goes beyond just the absence of these properties. We call a manifold $X$ totally non-Wecken if, for any integer $m$, there is a map $f_{m}: X \rightarrow X$ such that $M F\left[f_{m}\right]-N\left(f_{m}\right) \geq$ $m$. If the manifold $X$ has non-empty boundary, then in the same way we define $X$ to be totally non- $b W$ if, for any $m$, there is a boundary-preserving map $f_{m}$ with $M F_{\partial}\left[f_{m}\right]-N_{\partial}\left(f_{m}\right) \geq m$. It follows from a result of Kelly ([K2], Theorem 1.1) that the 2-manifolds with boundary of negative euler characteristic (with possibly a finite number of exceptions) not only fail to have the Wecken property but are in fact totally non-Wecken. In $\S 3$, we will show that if $X=$ $S \backslash\left(D_{1} \cup D_{2} \cup \cdots \cup D_{r}\right)$ is the 2-manifold obtained by removing $r \geq 1$ disjoint open discs $D_{j}$ from a closed 2-manifold $S$, then $X$ is totally non-bW if $S$ is not in the following list: sphere, projective plane, torus, Klein bottle, connected sum of three projective planes. In addition, the torus minus two or more discs is also totally non-bW.

Thus the possibilities for 2-manifolds with boundary which might behave differently in terms of the Wecken and the bW properties are quite limited. In $\S 4$, we carry out a detailed analysis of the fixed point behavior of the homotopy classes of boundary-preserving maps of one such 2-manifold: the sphere with three open discs removed, often called the "pants surface" $P$. We show that although $P$ is totally non-Wecken, it at least comes very close to the bW property. For maps $f:(P, \partial P) \rightarrow(P, \partial P)$, except for a few exceptional cases, we prove that $M F_{\partial}[f]=N_{\partial}(f)$. For the remaining cases, we can 
show that $M F_{\partial}[f] \leq N_{\partial}(f)+1$ and thus $P$ is almost $b W$ in the sense that $M F_{\partial}[f]-N_{\partial}(f)$ is bounded (by 1 ) for all $f$.

The techniques employed in $\S 4$ are of independent interest. In many of the proofs, our approach is to use methods of transversality theory to show that a map of the type being considered can be homotoped to one that is in a convenient standard form. It is then possible to describe explicit constructions for further homotoping the map, to one with only the relative Nielsen number of fixed points.

We demonstrate in $\S 4$ that the Wecken and bW properties are not identical in the 2-manifold setting, but we do not succeed in characterizing the bW property for all 2-manifolds with boundary. Therefore, in $\S 5$ we discuss the problems that remain.

2. Disc, annulus and Möbius band. Throughout the paper, given a boundary-preserving map $f:(X, \partial X) \rightarrow(X, \partial X)$ of a surface, we will denote the restriction of $f$ to the boundary by $\bar{f}: \partial X \rightarrow \partial X$.

In this section, we show that the three surfaces with boundary that have non-negative euler characteristic, that is, the ones listed in the title of the section, are bW.

(a) The disc. We view the disc $D$ as the unit disc in the complex plane with boundary $\partial D=C$. For a boundary-preserving map $f:(D, C) \rightarrow(D, C)$, if $\bar{f}$ is of degree $d \neq 1$, then it is homotopic to the map $\varphi_{d}: C \rightarrow C$ given by $\varphi_{d}(z)=z^{d}$ if $d \geq 0$ and $\varphi_{d}(z)=\bar{z}^{|d|}$ if $d<0$. The map $\varphi_{d}$ is of degree $d$ and has $|d-1|$ fixed points. Then $f$ is homotopic to a map $g:(D, C) \rightarrow(D, C)$ whose restriction to $C$ is $\varphi_{d}$ and that has no fixed points in the interior of the disc. (See $\S 2$ of $[\mathbf{B G}]$.) Since $N(\bar{f}) \leq N_{\partial}(f)$, we have shown that $M F_{\partial}[f]=N_{\partial}(f)$ when $d \neq 1$. In the case $d=1$, it is easy to see that $f$ may be homotoped to a map with $N_{\partial}(f)=1$ fixed point.

(b) The annulus. Write the boundary of the annulus $A$ as $\partial A=$ $C_{0} \cup C_{1}$ where the $C_{i}$ are circles. For a map $f:(A, \partial A) \rightarrow(A, \partial A)$, denote the restriction of $f$ to $C_{i}$ in the form $f_{i}: C_{i} \rightarrow C_{i^{*}}$.

LEMMA 2.1. If $f, g:(A, \partial A) \rightarrow(A, \partial A)$ are maps such that $\bar{f}, \bar{g}: \partial A \rightarrow \partial A$ are homotopic, then $f$ and $g$ are homotopic as maps of pairs.

Proof. Let $\Sigma$ be the "spindle-shaped" subset of $A \times I$ defined by $\Sigma=(A \times\{0,1\}) \cup\left(C_{0} \times I\right)$. By hypothesis, there is a homotopy between 
$f_{0}$ and $g_{0}$. Since $\Sigma$ is a strong deformation retract of $A \times I$, there is an extension of that homotopy to a homotopy $H: A \times I \rightarrow A$ between $f$ and $g$. Of course, $H$ might not take $C_{1} \times I$ to $C_{1^{*}}$. Therefore, we let $T$ be the subset of $A \times I \times I$ defined by

$$
T=(A \times I \times\{0\}) \cup(\Sigma \times I) \cup\left(C_{1} \times I \times I\right) .
$$

Let $r: A \times I \rightarrow A$ be a strong deformation retraction of $A$ onto $C_{1^{*}}$. A map from $T$ to $A$ may be defined by letting it be $H$ on $A \times I \times\{0\}$ and on each level of $\Sigma \times I$, and on $C_{1} \times I \times I$ it is the composition $r\left(\left(H \mid C_{1} \times I\right) \times 1_{I}\right)$. By the Homotopy Extension Theorem, this map extends to a map $\Gamma: A \times I \times I \rightarrow A$. The restriction of $\Gamma$ to $A \times I \times\{1\}$ is a homotopy between $f$ and $g$ as maps of pairs.

\section{THEOREM 2.2. The annulus is $b W$.}

Proof. Let $p_{0}: A=C_{0} \times I \rightarrow C_{0}$ be projection and note that $p_{0} f$ may be viewed as a homotopy between $p_{0} f_{0}$ and $p_{0} f_{1}$, so choosing orientations of the $C_{i}$ to agree with a chosen orientation of $A$, the maps $f_{0}: C_{0} \rightarrow C_{0^{*}}$ and $f_{1}: C_{1} \rightarrow C_{1^{*}}$ are of the same degree, call it $d$ and assume for now that $d \neq 1$. If $\left(0^{\#}, 1^{\#}\right)=(0,1)$, it follows that $N_{\partial}(f) \geq N(\bar{f})=2|d-1|$. We let $g(z, t)=\left(\varphi_{d}(z), t^{2}\right)$ for $(z, t) \in C \times I$, for $\varphi_{d}$ as in part (a), then $g$ has $2|d-1|$ fixed points for any value of $d$. The fact that $g$ is homotopic to $f$ as a map of pairs follows from the lemma. If $\left(0^{\#}, 1^{\#}\right)=(1,0)$, then $N(\bar{f})=0$ so $N_{\partial}(f)=N(f)=|d-1|$ and in this case we define $g(z, t)=\left(\varphi_{d}(z), 1-t\right)$. If $\left(0^{\#}, 1^{\#}\right)=(j, j), j$ either 0 or 1 , then $N_{\partial}(f) \geq N(\bar{f})=N\left(f_{j}\right)=|d-1|$ and letting $g(z, t)=\left(\varphi_{d}(z), j\right)$, we see that $g$ has $N_{\partial}(f)$ fixed points. Since $\bar{g}$ is homotopic to $\bar{f}$, the lemma completes the argument. The case $d=1$ is left to the reader.

(c) The Möbius band. We denote the Möbius band by $M$ and picture it as a triangle with vertices $v_{0}, v_{1}$ and $v_{2}$, with the edges oriented as $\left[v_{0}, v_{1}\right]\left[v_{1}, v_{2}\right]$ and $\left[v_{0}, v_{2}\right]$. Identify $\left[v_{0}, v_{1}\right]$ to $\left[v_{1}, v_{2}\right]$ and call the corresponding embedded curve $a$. After identification, the edge $\left[v_{0}, v_{2}\right]$ is a curve we call $b$. We have given $M$ the structure of a cell complex with 1-skeleton $a \cup b$. In the proof of the lemma that follows, we continue to use the maps $\varphi_{d}: C \rightarrow C$ and we need the obvious maps $a, b: S^{1} \rightarrow M$. These maps and their homotopy classes will be based. 
The key to the proof that the Möbius band is bW is the following result, which establishes for the Möbius band the property that Lemma 2.1 gives us for the annulus.

LEMMA 2.3. If $f, g:(M, \partial M) \rightarrow(M, \partial M)$ are maps such that $\bar{f}, \bar{g}: \partial M \rightarrow \partial M$ are homotopic, then $f$ and $g$ are homotopic as maps of pairs.

Proof. We allow confusion between maps and homotopy classes. Noting that $a \varphi_{2}=b$, we have $a^{-1} b=\varphi_{2}$. By hypothesis, the maps $f b$ and $g b$ are homotopic, so they are of the same degree, call it $d$. Then $f b=g b=b \varphi_{d}$. For the maps $f a$ and $g a$ we can find maps $K(f), K(g): S^{1} \rightarrow S^{1}$ such that $f a=a K(f)$ and $g a=a K(g)$. Therefore, up to homotopy, we may write

$$
\varphi_{2} \varphi_{d}=a^{-1} b \varphi_{d}=a^{-1} f b=K(f) a^{-1} b=K(f) a^{-1} a \varphi_{2}=K(f) \varphi_{2}
$$

which implies that $K(f)$ is of degree $d$. But the same argument works for $g$ as well, so $K(g)$ is also of degree $d$ and we conclude that $f a$ and $g a$ are (based) homotopic. Thus $f$ and $g$ are homotopic on the one-skeleton $a \cup b$ of $M$. But $\pi_{2}(M)=0$ so $f$ and $g$ are in fact homotopic as maps of $(M, \partial M)$.

\section{THeORem 2.4. The Möbius band is $b W$.}

Proof. For each integer $d$, we will exhibit a map $f_{d}:(M, \partial M) \rightarrow$ $(M, \partial M)$ such that $\bar{f}_{d}: \partial M \rightarrow \partial M$ is of degree $d$ and $f_{d}$ has $N_{\partial}\left(f_{d}\right)$ fixed points. This will prove the theorem since, given a map $f:(M, \partial M) \rightarrow(M, \partial M)$ and letting $d$ be the degree of $\bar{f}$, then Lemma 2.3 implies that $f$ is homotopic to that $f_{d}$ as a map of pairs and it further follows from [S] that $N_{\partial}(f)=N_{\partial}\left(f_{d}\right)$. If $d$ is an even integer, we write $d=2 k$. The maps $a, b: S^{1} \rightarrow M$ orient the curves $a\left(S^{1}\right)$ and $b\left(S^{1}\right)$ which we abbreviate as $a$ and $b$, respectively, and it makes sense to define $\varphi_{k}: a \rightarrow b$. We can further retract $M$ onto $a$ in such a way as to identify the restriction of the retraction $r$ to $\partial M=b$ with $\varphi_{-2}: b \rightarrow a$. We define $f_{d}=\varphi_{k} r$ and note that the restriction of $f_{d}$ to $b$ is $\varphi_{d}$. Since $f_{d}$ maps $M$ onto $\partial M$, we see that $f_{d}$ has $|d-1|=N\left(\bar{f}_{d}\right)=N_{\partial}\left(f_{d}\right)$ fixed points, as required. When $d$ is odd, we need slightly different constructions of $f_{d}$ depending on whether $d$ is positive or negative. The case $d=1$ is easily dealt with by taking $f_{d}$ to be a fixed point free map. For the case $d=2 k+1>1$, 


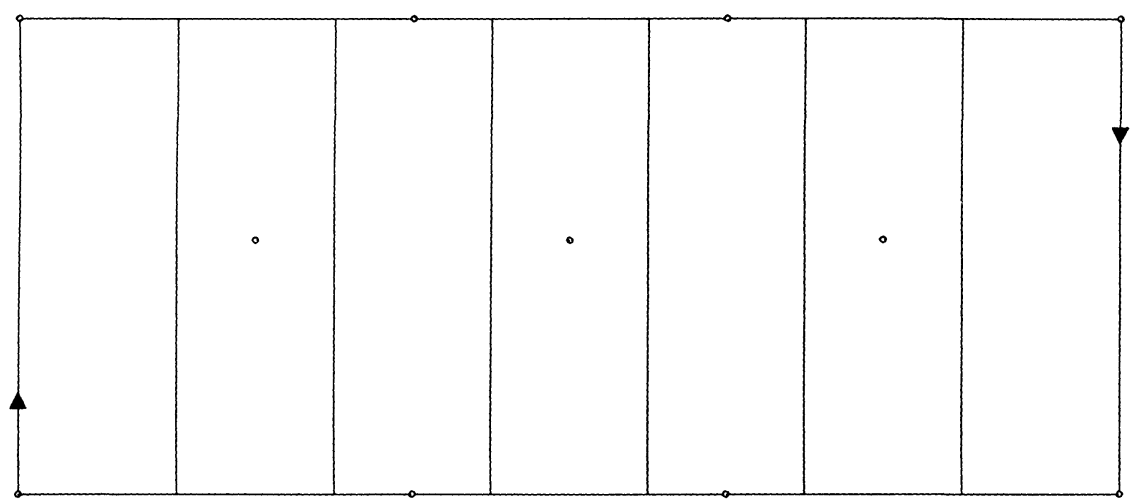

FIGURE 1

we let $f_{d}$ be the $2 k+1$-fold covering map; pictured in Figure 1 for the case $k=3$.

The left-hand rectangle is stretched over the entire Möbius band and the left-hand edge perturbed slightly so that there are only two fixed points, as indicated in the figure, where those points reappear on the right side in the reversed order. The next rectangle is flipped over as well as stretched over $M$, so it has only the single fixed point indicated. The third rectangle behaves like the first one: there is a vertical interval of fixed points which can be reduced to two by a perturbation of the interior of the interval. Continuing in this way, we obtain the map $f_{d}=f_{2 k+1}$ with $2 k$ fixed points in $\partial M$ and $k$ fixed points in the interior of $M$. Since both $f_{d}$ and $\bar{f}_{d}$ are generically $d$ to-one maps, they are of degree $d$ and so $N\left(f_{d}\right)=N\left(\bar{f}_{d}\right)=|d-1|=$ $2 k$. It is clear from the construction that when two fixed points lie in the same rectangle, they are Nielsen equivalent, so at most $k$ of the fixed point classes of $f_{d}$ are represented on $\partial M$ and therefore each of the $k$ fixed points on the interior of $M$ must be a different essential fixed point class of $f_{d}$. We conclude that $N_{\partial}\left(f_{d}\right)=3 k$ and therefore $f_{d}$ has the required properties in this case. Finally we suppose that $d=1-2 k$ where $k \geq 1$ and we define $f_{d}$ as a $|1-2 k|$-fold cover. The definition is similar to that of the last case, except that each rectangle is reflected about a vertical line segment dividing it in half before it is mapped to $M$. For the left-most rectangle this reverses the points at the "corners" that were fixed in the previous case and instead we have a fixed point at the center of the interval, as indicated. Now, since the top segment of that rectangle is reversed before mapping to the top of $M$, it must contain a fixed point. The bottom segment also must contain a fixed point, which is obviously in the same fixed point 


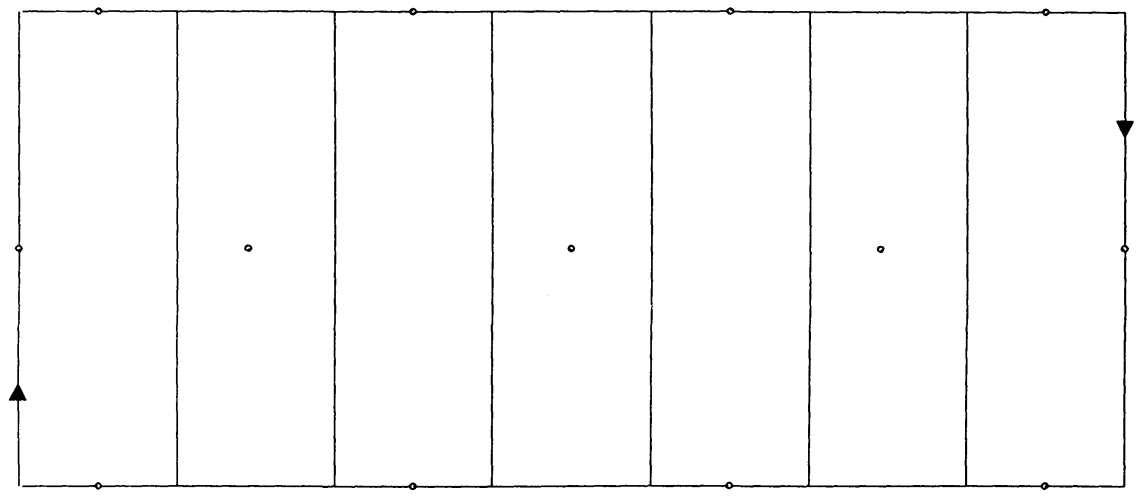

FIGURE 2

class as the one on the top. In the next rectangle the top and bottom segments are still reversed, so we have just a single fixed point, as in the case when $d$ was positive. As Figure 2 shows us in the $k=4$ case, there are thus $2 k$ fixed points on $\partial M$ and another $k$ fixed points in the interior. Since again $N\left(f_{d}\right)=N\left(\bar{f}_{d}\right)=|d-1|=2 k$ and there are at most $k$ fixed point classes of $f_{d}$ appearing in $\partial M$ we have $N_{\partial}\left(f_{d}\right)=3 k$ in this case also.

\section{Totally non-bW surfaces.}

THEOREM 3.1. Let $X$ be the surface obtained in one of the following ways: (i) deleting $r \geq 2$ open discs from the torus, (ii) deleting $r \geq 1$ open discs from the connected sum of two or more tori, (iii) deleting $r \geq 1$ open discs from the connected sum of four or more projective planes. Given an integer $m>\frac{r}{2}$ there exists a map $f:(X, \partial X) \rightarrow$ $(X, \partial X)$ such that $N_{\partial}(f)=r$ and $M F_{\partial}[f] \geq 2 m$. Therefore, $X$ is totally non- $b W$.

Proof. We first construct a map $f^{\prime}: X \rightarrow X$. The surface $X$ can be projected onto the plane as shown in Figure 3 (see next page). In case (i) the broken handle is not present. In the other cases it is present and may be twisted, and there may be additional handles, some of which may be twisted, attached to the dotted region in the figure. Assume for now that we are in case (ii) or (iii). Then $\partial X=C \cup C_{1} \cup \cdots \cup C_{r-1}$ where $C$ denotes the "outside" boundary component. The loop $\alpha$ intersects each $C_{j}$ for $j=1, \ldots, r-1$ in a point $x_{j}$. There is a "pants surface" $P$ (disc with two holes) imbedded in $X$, containing the loops $\alpha$ and $\beta$ with part of $\alpha$ on the boundary of $P$, as indicated 


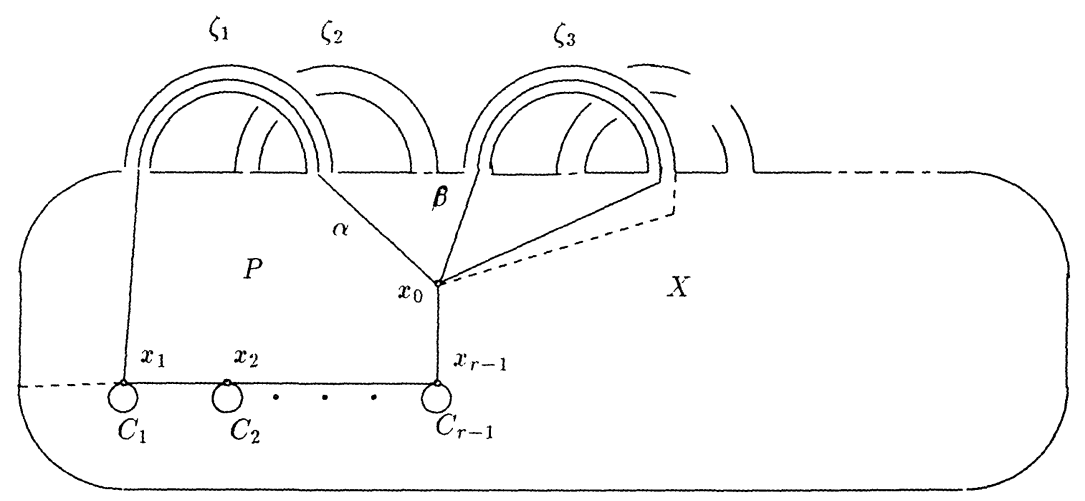

Figure 3

by the dashed lines in the figure. Let $\rho: X \rightarrow \alpha$ be a retraction such that the loop $\beta$ is taken to an arc in $\alpha$ and $\rho\left(C_{j}\right)=x_{j}$. Define a map $\eta: \alpha \rightarrow \alpha \cup \beta$ in the following way. Send the arc in $\alpha$ from $x_{0}$ to $x_{1}$ to itself by the identity map. Then send the rest of $\alpha$ to $\alpha \cup \beta$ so that, as an element of $\pi_{1}\left(X, x_{0}\right)$, we have $\alpha$ sent to the word $[\beta, \alpha]^{m} \beta \alpha$, where $[\beta, \alpha]$ denotes the commutator $\beta \alpha \beta^{-1} \alpha^{-1}$. Let $f^{\prime}=i \eta \rho: X \rightarrow X$, where $i$ is the inclusion of $\alpha \cup \beta$ in $X$. By the commutativity property of the Nielsen number, $N\left(f^{\prime}\right)=N(\rho(i \eta))=0$ since $\rho(i \eta): \alpha \rightarrow \alpha$ is of degree one.

The map $f^{\prime}: X \rightarrow X$ is not boundary-preserving because the boundary component $C$ does not go into $\partial X$, so we must next modify the definition to obtain this property. We note that $\rho \mid C: C \rightarrow X$, the restriction of the retraction to $C$, is an inessential map. To demonstrate this fact, choose a point $x^{*}$ on $\alpha$ that lies in the handle through which $\alpha$ passes. Then $(\rho \mid C)^{-1}\left(x^{*}\right)$ consists of two points on the boundary of the handle, and $\rho \mid C$ is of opposite degrees at these points, so the degree of $\rho \mid C$ is zero. We conclude that $f^{\prime}|C=i \eta \rho| C$ is also inessential. Therefore, there is a homotopy of $f^{\prime} \mid C$ to a constant map taking $C$ to a point $x_{r}$ and thus a homotopy of the restriction of $f^{\prime}$ to $\partial X$ to a map taking each boundary component to a point $x_{j}$, for $j=$ $1, \ldots, r$, on that component. By the Homotopy Extension Theorem, therefore, we can produce a map $f:(X, \partial X) \rightarrow(X, \partial X)$ homotopic to $f^{\prime}$. Since $N(f)=N\left(f^{\prime}\right)=0$, it follows that $N_{\partial}(f)=N(\bar{f})=r$.

We will show that $M F\left[f^{\prime}\right]=2 m$ and since clearly $M F_{\partial}[f] \geq$ $M F[f]=M F\left[f^{\prime}\right]$, this will complete the proof of Theorem 3.1. The map $f^{\prime}$ was defined so that its restriction to the pants $f^{\prime} \mid P: P \rightarrow P$ is the map $g_{m}$ of Corollary 1.2 of [K1] which, according to that result, 
has the property $M F\left[g_{m}\right]=2 m$. Since $P$ is a retract of $X$, the inclusion $i: P \rightarrow X$ induces a monomorphism of the fundamental groups. The map $f^{\prime}$ was constructed so that its image is $\alpha \cup \beta$, a subset of $P$. We clearly have that $i\left(f^{\prime} \mid P\right)=f^{\prime} i$, so the hypotheses of Theorem 1.1 of [K2] are satisfied, and we conclude that $M F\left[f^{\prime}\right]=$ $M F\left[f^{\prime} \mid P\right]=2 m$. In case (i), there are only three handles, denoted in Figure 3 by $\zeta_{1}, \zeta_{2}, \zeta_{3}$, and there are $r-2$ curves $C_{j}$ because the handle $\zeta_{3}$ determines a boundary component. Since $f^{\prime}(\beta)$ is contractible, we may still homotope $f^{\prime}$ to a boundary preserving map and complete the proof as in the other cases.

4. The pants surface. Let $P$ denote the disc with two holes, known informally as the "pants surface". It was proved in [K1] that $P$ is totally non-Wecken: for any integer $m \geq 1$ there is a map $f_{m}: P \rightarrow P$ such that $M F\left[f_{m}\right]-N\left(f_{m}\right) \geq m$. In this section, we will show that with respect to the fixed point theory of boundary-preserving maps, the surface $P$ behaves very differently: $M F_{\partial}[f]-N_{\partial}(f) \leq 1$ for any map $f:(P, \partial P) \rightarrow(P, \partial P)$.

The components of its boundary, $\partial P$, are written as $C_{0}, C_{1}$, and $C_{2}$. For a map $f:(P, \partial P) \rightarrow(P, \partial P)$, we continue to write $f_{j}: C_{j} \rightarrow C_{j^{*}}$ for the restriction of $f$ to each boundary component, just as we did for maps of the annulus.

Assume that $P$ is embedded in the plane so that $C_{1}$ and $C_{2}$ are contained in the bounded component of the complement of $C_{0}$. Choose a clockwise orientation for $C_{1}$ and $C_{2}$ and a counterclockwise orientation for $C_{0}$. Select a base point $x_{0} \in C_{0}$ and $\operatorname{arcs} \omega_{j}$ for $j=1,2$, each with one endpoint at $x_{0}$ and the other at a point $x_{j}$ in $C_{j}$, see Figure 4 on next page.

Define loops $\sigma_{j}$ at $x_{0}$ by $\sigma_{j}=\omega_{j} C_{j} \omega_{j}^{-1}$; then we may view $\pi_{1}\left(P, x_{0}\right)$ as the free group generated by $\left[\sigma_{1}\right]$ and $\left[\sigma_{2}\right]$. Set $\left[\sigma_{0}\right]=$ $\left[C_{0}\right]$ and note that $\left[\sigma_{1}\right]\left[\sigma_{2}\right]=\left[\sigma_{0}\right]^{-1}$ in $\pi_{1}\left(P, x_{0}\right)$.

Proposition 4.1. Suppose $f:(P, \partial P) \rightarrow(P, \partial P)$ is a map such that $f_{j}$ is essential for all $j=0,1,2$. If $g:(P, \partial P) \rightarrow(P, \partial P)$ is homotopic to $f$ as a map from $P$ to itself and $\bar{g}: \partial P \rightarrow \partial P$ is homotopic to $\bar{f}$, then $g$ is homotopic to $f$ as a map of pairs.

Proof. Let $H^{[-1]}: P \times I \rightarrow P$ be a homotopy between $f$ and $g$, the existence of which is given by hypothesis. We will construct homotopies $H^{[j]}$ for $j=0,1,2$ between $f$ and $g$ such that $H^{[j]}$ maps $C_{i} \times I$ into $C_{i^{*}}$ for all $i \leq j$ and therefore $H^{[2]}$ will be the 


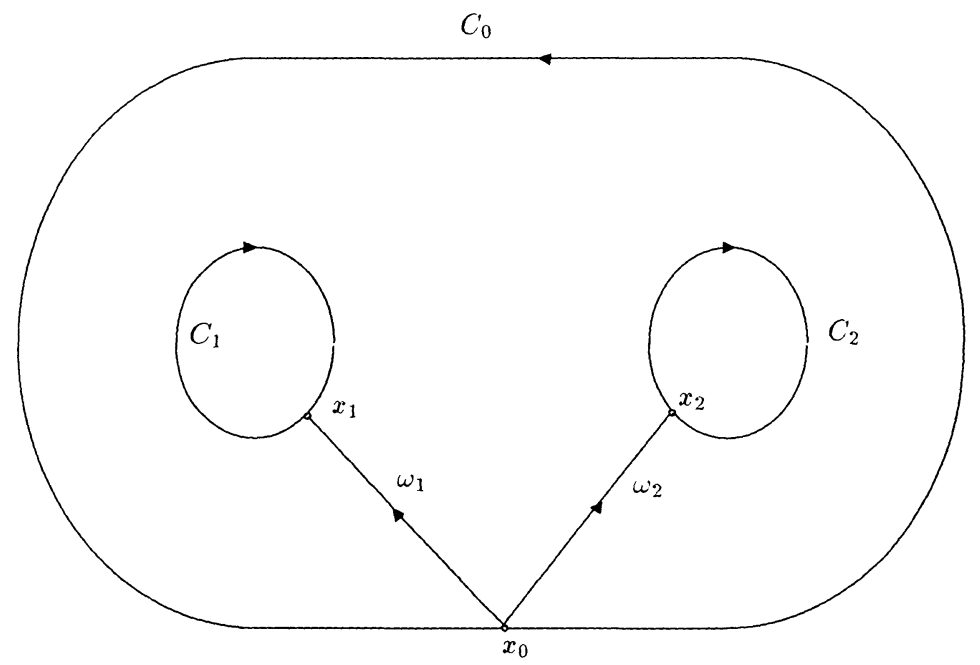

FIGURE 4

required homotopy of pairs. Thus we assume $H^{[j-1]}$ has been constructed and let $\eta: C_{j} \times I \rightarrow P$ be the restriction of $H^{[j-1]}$. Choose an arc $a$ in $P$ that meets $\partial P$ only in its endpoints, one in each component of $\partial P$ other than $C_{j^{*}}$. Noting that, by hypothesis, $f$ and $g$ map $C_{j}$ to $C_{j^{*}}$, we make $\eta$ transverse to $a$ rel $C_{j} \times\{0,1\}$ so that $\eta^{-1}(a)$ is a union of simple closed curves in the interior of $C_{j} \times I$. Imbed $C_{j} \times I$ in the plane so that $C_{j} \times\{0\}$ lies in the bounded component of the complement of $C_{j} \times\{1\}$. Let $K$ be a component of $\eta^{-1}(a)$; then, by the Schönflies Theorem, the closure of one of the components of the complement of $K$ is a disc $D$. If $D$ contained $C_{j} \times\{0\}$, then $K$ and $C_{j} \times\{0\}$ would bound an annulus and $\eta \mid K$ and $\eta\left|\left(C_{j} \times\{0\}\right)=f\right| C_{j}=f_{j}$ would be homotopic. But $\eta(K)$ is contained in the arc $a$ whereas, by hypothesis, $f_{j}$ is an essential map of $C_{j}$, onto $C_{j^{*}}$, which is freely homotopic, and therefore conjugate in $\pi_{1}\left(P_{r}, x_{0}\right)$, to a nontrivial element of that group. We conclude that $D$ does not contain $C_{j} \times\{0\}$ but instead lies entirely in $C_{j} \times I$ and thus $K$ is inessential. Since $\eta^{-1}(a)$ is a union of inessential simple closed curves, we may use an innermost circle argument to homotope $\eta\left(\right.$ rel $\left.C_{j} \times\{0,1\}\right)$ so that the image is disjoint from $a$. Furthermore, since $C_{j^{*}}$ is a strong deformation retract of $P \backslash a$, we have a homotopy $h: C_{j} \times I \times I \rightarrow P$ between $\eta$ and a map that takes $C_{j} \times I$ to $C_{j^{*}}$. Let $T$ be the subset of $P \times I \times I$ which is the union of $P \times I \times\{0\}$, $P \times\{0,1\} \times I$ and all $C_{i} \times I \times I$ for $i \leq j$. Define a map from $T$ to $P$ to be $H^{[j-1]}$ on $P \times I \times\{0\}$ and $C_{i} \times I \times\{t\}$ for all $t$, for $i<j$, 


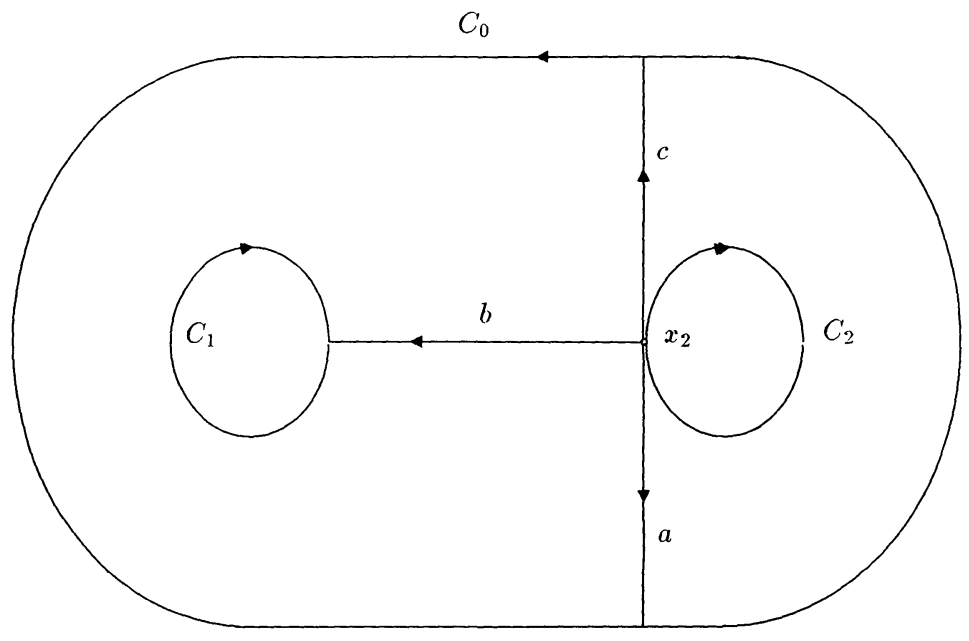

FIGURE 5

to be $f$ and $g$ on $P \times\{0\} \times\{t\}$ and $P \times\{1\} \times\{t\}$, respectively, for all $t$, and to be $h$ on $C_{j} \times I \times I$. Extend the map to $\Gamma: P \times I \times I \rightarrow P$ by the Homotopy Extension Theorem and the required homotopy is the restriction of $\Gamma$ to $P \times I \times\{1\}$ (compare Lemma 2.1).

Remark. Proposition 4.1 is false if the $f_{j}$ are not essential. Consider maps which are constant on each component of $\partial C$, constant outside collars of $C_{0}$ and $C_{1}$ and constant on each parallel circle within the collars. The images of two such maps $f$ and $g$ are illustrated in Figure 5 where $f(P)=a \cup b, g(P)=b \cup c$ and both $f$ and $g$ preserve boundary components. These maps are clearly homotopic. Suppose they were homotopic as maps of pairs. From now on we only consider $f$ and $g$ on $a \cup b$. We can assume that $f$ is the identity on $a \cup b$ and $g$ is the identity on $b$, and $g(a)=c$. Now change $g$, by a homotopy, so that $g$ is given by $b \mapsto b, a \mapsto C_{2} a$. Let $H: f \simeq g$ on $a \cup b$. The track of $x_{2}$ under this homotopy is a loop in $C_{2}$. Now the loop once around $C_{2}$ is homotopic in $P$ to the loop given by $b C_{1}^{-1} b^{-1} a C_{0}^{-1} a^{-1}$ so we can get a homotopy rel $x_{2}$ between a modification of the identity given by:

$$
\begin{aligned}
& a \mapsto\left(b C_{1}^{-1} b^{-1} a C_{0}^{-1} a^{-1}\right)^{k} a, \\
& b \mapsto\left(b C_{1}^{-1} b^{-1} a C_{0}^{-1} a^{-1}\right)^{k} b \text { for some } k
\end{aligned}
$$

and the map $g$ given by $a \mapsto C_{2} a, b \mapsto b$. Now this homotopy can be assumed to take place in $C_{0} \cup a \cup b \cup C_{1}$ since this space is a strong deformation retract of $P$. By collapsing $b \cup C_{1}$ and looking at the 
maps on $a$ we see that $k=1$, but by collapsing $a \cup C_{0}$ and looking at the maps on $b$ we get $k=0$.

Given a map $f:(P, \partial P) \rightarrow(P, \partial P)$, we can homotope $f$ as a map of pairs so that $f\left(x_{j}\right)=x_{j^{*}}$ for $j=0,1,2$. The maps $f_{j}: C_{j} \rightarrow C_{j^{*}}$ are of degrees $d_{j}$ with respect to the given orientations.

LEMMA 4.2. Let $f_{\pi}: \pi_{1}\left(P, x_{0}\right) \rightarrow \pi_{1}\left(P, x_{0}\right)$ be induced by a map $f:(P, \partial P) \rightarrow(P, \partial P)$. Then $f_{\pi}\left[\sigma_{j}\right]$ is conjugate to $\left[\sigma_{j^{*}}\right]^{d}$, for $j=$ $0,1,2$.

Proof. Since $f_{j}$ is of degree $d_{j}$, then

$$
\left[\omega_{j^{*}} f\left(C_{j}\right) \omega_{j^{*}}^{-1}\right]=\left[\omega_{j^{*}}\left(C_{j^{*}}\right)^{d} \omega_{j^{*}}^{-1}\right]
$$

in $\pi_{1}\left(P, x_{0}\right)$, and therefore

$$
\begin{aligned}
f_{\pi}\left[\sigma_{j}\right] & =\left[f\left(\omega_{j} C_{j} \omega_{j}^{-1}\right)\right] \\
& =\left[f\left(\omega_{j}\right) \omega_{j^{*}}^{-1}\right]\left[\omega_{j^{*}} f\left(C_{j}\right) \omega_{j^{*}}^{-1}\right]\left[\omega_{j^{*}} f\left(\omega_{j}^{-1}\right)\right] \\
& =\left[f\left(\omega_{j}\right) \omega_{j^{*}}^{-1}\right]\left[\omega_{j^{*}}\left(C_{j^{*}} d_{\lrcorner} \omega_{j^{*}}^{-1}\right]\left[\omega_{j^{*}} f\left(\omega_{j}^{-1}\right)\right]\right. \\
& =\left[f\left(\omega_{j}\right) \omega_{j^{*}}^{-1}\right]\left[\sigma_{j^{*}}\right]^{d}\left[\omega_{j^{*}} f\left(\omega_{j}^{-1}\right)\right] .
\end{aligned}
$$

For a map $f:(P, \partial P) \rightarrow(P, \partial P)$, let $\operatorname{Im}_{\partial}(f)$ denote the number of components of $\partial P$ that contain points of $f(\partial P)$.

LEMMA 4.3. Let $f:(P, \partial P) \rightarrow(P, \partial P)$ be a map such that $\operatorname{Im}_{\partial}(f)$ $=3$; then all the maps $f_{j}: C_{j} \rightarrow C_{j^{*}}$, for $j=0,1,2$, are of the same degree, $d$.

Proof. We can make $P$ into a 2 -sphere by attaching discs $D_{j}$ along the boundary components $C_{j}$. The map $f$ then extends to a map $g: S^{2} \rightarrow S^{2}$ by using the fact that each $D_{j}$ is a cone on $C_{j}$. After an excision we see that the induced homomorphism $g_{*}: H_{2}\left(D_{j}, C_{j}\right) \rightarrow$ $H_{2}\left(D_{j^{*}}, C_{j^{*}}\right)$ is given by multiplication by $\operatorname{deg}(g)$. The homomorphism extends to a homomorphism of exact sequences of pairs. From this we see that $\operatorname{deg}(g)=\operatorname{deg}\left(f_{j}\right)$.

LEMMA 4.4. Let $f:(P, \partial P) \rightarrow(P, \partial P)$ be a map such that $\operatorname{Im}_{\partial}(f)$ $=3$; then $|d| \leq 1$. 

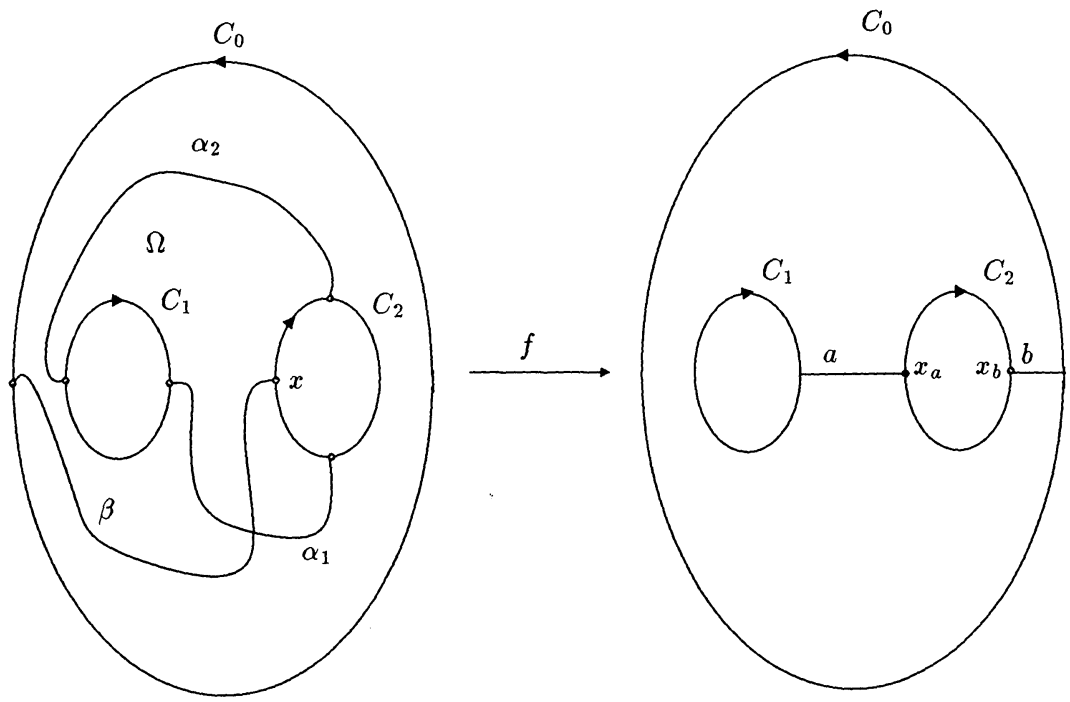

FIGURE 6

Proof. We may assume without loss of generality that $f$ maps each $C_{j}$ to itself and that each $f_{j}$ can be identified with a map $\varphi_{d}$ from $\S 2$. By the preceding lemma, the degree $d$ is the same for all $j$. Let $a$ and $b$ be arcs connecting $C_{1}$ to $C_{2}$ and $C_{2}$ to $C_{0}$, respectively, and make $f$ transverse to $a \cup b$. (See Figure 6.) Now assume that $|d| \geq 2$.

By transversality, $f^{-1}(a)$ contains $|d|$ arcs connecting a point of $C_{1}$ to a point of $C_{2}$; let $\alpha_{1}$ and $\alpha_{2}$ be any two of them. Let $x_{a}$ and $x_{b}$ be the intersection of $C_{2}$ with $a$ and $b$, respectively. The $|d|$ points of $f_{2}^{-1}\left(x_{a}\right)$ alternate with the $|d|$ points of $f_{2}^{-1}\left(x_{b}\right)$. The arcs $\alpha_{1}$ and $\alpha_{2}$ together with properly chosen arcs of $C_{1}$ and $C_{2}$ bound a region $\Omega$ in $P$. Let $x \in C_{2}$ be a point of $f_{2}^{-1}\left(x_{b}\right)$ that is on the boundary of $\Omega$; then there is an arc $\beta$ of $f^{-1}(b)$ connecting $x$ to a point of $C_{0}$. The arc $\beta$ must contain points of $\Omega$, yet $C_{0}$ is in the unbounded complementary domain of $\Omega$, so the Jordan Curve Theorem implies, since $\beta$ is contained in $P$, that $\beta$ must intersect $\alpha_{1}$ or $\alpha_{2}$, and that is impossible since $a$ and $b$ are disjoint. We conclude that $|d| \leq 1$.

LEMMA 4.5. If $f:(P, \partial P) \rightarrow(P, \partial P)$ is a map with $\operatorname{Im}_{\partial}(f)=3$ and $d \neq 0$, then $M F_{\partial}[f]=N_{\partial}(f)$.

Proof. We claim that the homomorphism $f_{\pi}: \pi_{1}\left(P, x_{0}\right) \rightarrow \pi_{1}\left(P, x_{0^{*}}\right)$ induced by $f$ is an isomorphism, and furthermore that there is a 
homeomorphism $h: P \rightarrow P$ that induces $f_{\pi}$. Assume first that $f$ maps $C_{j}$ to itself, for $j=0,1,2$, and that $d=1$. By Lemma 4.2, we may write $f_{\pi}\left[\sigma_{1}\right]=\alpha\left[\sigma_{1}\right] \alpha^{-1}$, and $f_{\pi}\left[\sigma_{2}\right]=\beta\left[\sigma_{2}\right] \beta^{-1}$. We may assume that these expressions have been reduced in the group $\pi_{1}\left(P, x_{0}\right)$. Certainly we have $f_{\pi}\left[\sigma_{0}\right]^{-1}=\left[\sigma_{0}\right]^{-1}$. Recalling that $\left[\sigma_{1}\right]\left[\sigma_{2}\right]=\left[\sigma_{0}\right]^{-1}$, we see that

$$
\alpha\left[\sigma_{1}\right] \alpha^{-1} \beta\left[\sigma_{2}\right] \beta^{-1}=\left[\sigma_{1}\right]\left[\sigma_{2}\right] .
$$

Therefore, the left-hand side must reduce and since we assumed the conjugations were already reduced, we conclude that $\alpha=\beta$. But the only word in $\pi_{1}\left(P, x_{0}\right)$ that is reduced when conjugated with both generators is the identity, so $f_{\pi}$ is the identity isomorphism, which is induced by a homeomorphism, the identity. Now let $f$ retain only the property that $d=1$, then there is an orientation-preserving homeomorphism $\theta$ of $P$ such that $\theta f$ takes each component of $\partial P$ to itself. By the first part of the proof, $\theta_{\pi} f_{\pi}$ is the identity and thus $f_{\pi}=\left(\theta_{\pi}\right)^{-1}=\left(\theta^{-1}\right)_{\pi}$ so $f_{\pi}$ is an isomorphism induced by a homeomorphism, $h=\theta^{-1}$. For the case that $d=-1$, we need only choose $\theta$ to be orientation-reversing, and this will complete the proof of the claim. The homeomorphism $h$ is homotopic to $f$ because $P$ is a $K(\pi, 1)$. By Lemma 4.2, we see that $h$ must take $C_{1}$ and $C_{2}$ to the same components of $\partial P$ as $f$ does. Recalling that the $f_{j}$ are all essential, we see that the hypotheses of Proposition 4.1 are satisfied and therefore $h$ is homotopic to $f$ as a map of pairs. By Theorem 5.1 of [JG], the homeomorphism $h$ is isotopic to a homeomorphism with exactly $N_{\partial}(h)=N_{\partial}(f)$ fixed points.

LEMMA 4.6. Let $f:(P, \partial P) \rightarrow(P, \partial P)$ be a map with $\operatorname{Im}_{\partial}(f)=$ 2. Of the maps $f_{j}: C_{j} \rightarrow C_{j^{*}}$, the sum of the degrees of the two that map to the same component of $\partial P$ is zero and the remaining map is inessential.

Proof. Let $C_{i^{*}}$ be the component of $\partial P$ to which one boundary component, $C_{i}$, is mapped by $f$. Then if we attach discs $D_{i}$ to the domain and $D_{i^{*}}$ to the range we have a map of an annulus to an annulus and the first part follows-project the image annulus to a circle. If as in the proof of 4.3 we extend to a map $g$ of a 2sphere then we can see that $g$ has degree zero, since $g$ is not onto. The result now follows by computing the degree in two ways-look at inverse images of the two discs which are in the image of $g$. 
Suppose that $f:(P, \partial P) \rightarrow(P, \partial P)$ is a map. When we want to be specific as to where boundary components go, we will say that $f$ is of type $\left(0^{\#}, 1^{\#}, 2^{\#}\right)$ to mean that $f_{j}: C_{j} \rightarrow C_{j^{*}}$ for $j=0,1,2$. Call $f$ boundary inessential if $f$ is null homotopic on each boundary component.

Now suppose $\operatorname{Im}_{\partial}(f)=2$ and $f$ is not boundary inessential. Suppose $\operatorname{im}(f) \cap C_{0}=\varnothing$, and $f\left(C_{0}\right)=C_{1}$; then up to numbering of the components of $\partial P$ there are three cases to be considered given by $(1,1,2),(1,2,1)$, and $(1,2,2)$.

THEOREM 4.7. If $f:(P, \partial P) \rightarrow(P, \partial P)$ is a map such that $\operatorname{Im}_{\partial}(f)$ $=2$ and $f$ is not boundary inessential, then $M F_{\partial}[f]=N_{\partial}(f)$.

Proof. Case $(1,1,2)$. We will find a homotopy of $f$ to a map which has no fixed points on the interior of $P$. Let $a$ be an arc intersecting $C_{1}$ and $C_{2}$ at $x_{1}$ and $x_{2}$, respectively, and otherwise disjoint from $\partial P$. Let $Z=C_{1} \cup a \cup C_{2}$; then there is a deformation retraction $r: P \rightarrow Z$ and we note that $r f$ is homotopic to $f$ as a map of pairs. By the Homotopy Extension Theorem we may assume that $f_{1}$ can be identified with the map $\varphi_{d}$, that we introduced in $\S 2$, with $f\left(x_{1}\right)=x_{1}$. Furthermore, by the preceding lemma, we may assume that $f_{2}$ is the constant map at $x_{2}$. Choose $b_{i}$ in $C_{i}$ for $i=1,2$ so that $b_{i} \neq x_{i}$ and choose $b_{3}$ in the interior of $a$. We now invoke the theory of transverse cw complexes of [BRS], Chapter 7. After a homotopy we can assume that $\operatorname{im}(f)=Z$ and $f$ is transverse to $Z$; in particular this means $Y=f^{-1}\left\{b_{1} \cup b_{2} \cup b_{3}\right\}$ is a 1-manifold, and inverse images of the 1-cells in $Z$ form a trivialised tubular neighourhood of $Y$. The regions between the components of the tubular neighbourhood get mapped to $\left\{x_{1}, x_{2}\right\}$. After a further homotopy rel $\partial P$, which will eliminate innermost circles, we can assume that $Y$ appears as in Figure 7 (see next page), except that the loop $h$ is not in $Y$.

The arc $a$ is now partitioned into intervals each of which is mapped into one of the following: $C_{1}, C_{2}, a, x_{1}, x_{2}$. Interior fixed points can now only occur in $a$. After a homotopy we can assume that neighbouring circles of $Y$ are not both mapped to $b_{3}$-they would represent a "fold" along $a$. Consider one of the subintervals of $a$ which is now mapped to $a$, it will lie between subintervals which are mapped to $\left\{x_{1}, x_{2}\right\}$. Change the partition of $a$ so that these three subintervals are now counted as one and labelled as $a$ if the map preserves orientation on this amalgamated interval and $a^{-1}$ otherwise. 


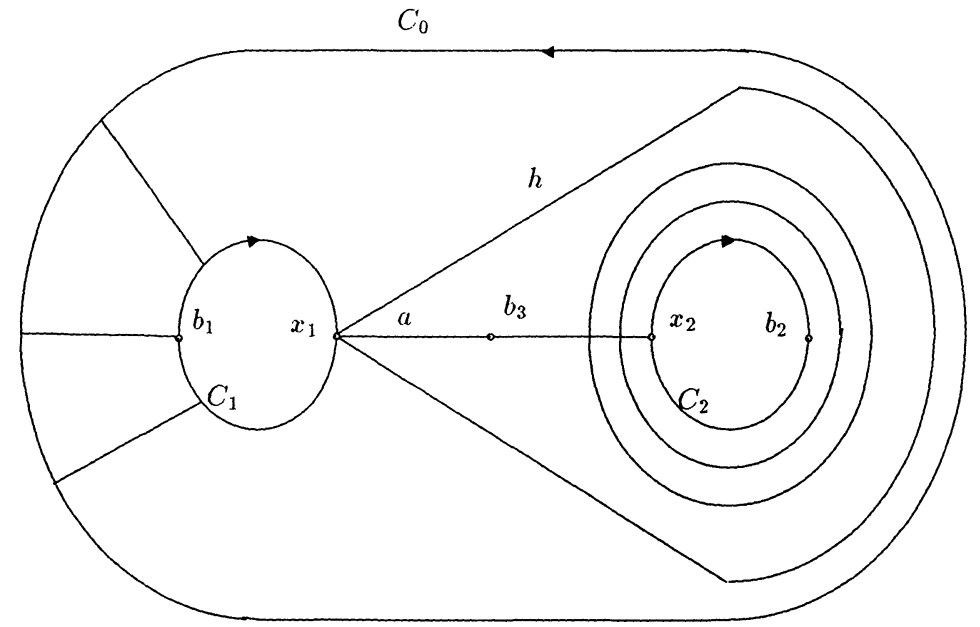

FIGURE 7

Similarly label the remaining intervals by $C_{i}$ or $C_{i}^{-1}$ for $i=1,2$. Reading from $x_{1}$ to $x_{2}$ along $a$ we can assume that we get a word of the form:

$$
C_{1}^{n_{1}} a C_{2}^{n_{2}} a^{-1} C_{1}^{n_{3}} a C_{2}^{n_{4}} a^{-1} \ldots C_{1}^{n_{r}} a .
$$

The word begins with $C_{1}^{n_{1}}$ because $f\left(x_{1}\right)=x_{1}$, but it may be that $n_{1}=0$. Otherwise we may assume all $n_{i} \neq 0$. Since $f\left(x_{2}\right)=x_{2}$, the word could end with $C_{2}^{n_{r+1}}$. However since $f$ is constant on $C_{2}$, we may deform the map so that the final $C_{2}$ term vanishes.

Let $h$ be a loop in $P$ based at $x_{1}$ and lying in the region outside the regular neighbourhood, so that $f(h)=x_{1}$. Over each triple of intervals of $a$ that corresponds to an expression $a C_{2}^{k} a^{-1}$, we deform that loop based at $x_{1}$ to the loop $h^{k}$. We still call our map $f$, though its image is now $Z \cup h$ and its effect on $a$ is now represented by the word:

$$
C_{1}^{n_{1}} h^{n_{2}} C_{1}^{n_{3}} h^{n_{4}} \ldots C_{1}^{n_{r}} a .
$$

The map $f$ has only the fixed point $x_{1}$ on $h$ since $f(h)=x_{1}$ and now the only interval of $a$ that is mapped to $a$ is the one containing $x_{2}$, which is stretched over $a$ and thus has a fixed point only at $x_{2}$. Thus $f$ has no fixed points on the interior of $P$. If $d \neq 1$, then $f$ has exactly $N(\bar{f})$ fixed points on $\partial P$, so certainly $M F_{\partial}[f]=N_{\partial}(f)$.

If $d=1$ then $f_{1}$ has $x_{1}$ as fixed point. We must modify $f$ so that it has no fixed points on $C_{1}$. To do this note that $C_{1}$ has a collar neighbourhood $N$ which is mapped to $C_{1}$. Change $f$ on $N$, keeping the outer boundary fixed, and so that $f$ on $C_{1}$ is a degree one map 


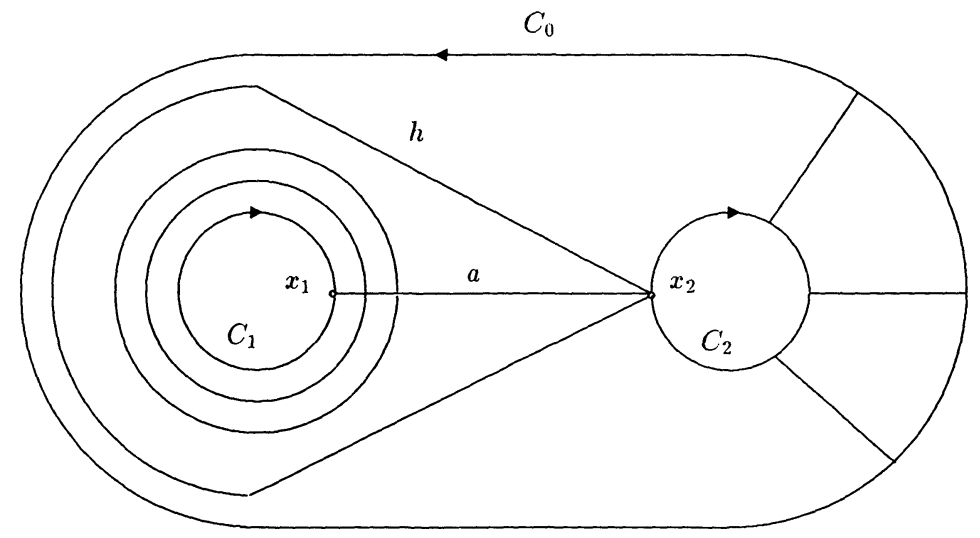

FIGURE 8

without fixed points. There is now only one fixed point in $P$, namely $x_{2}$.

Case $(1,2,1)$. In this case $Y$ is pictured in Figure 8 and the image of $a$ is given by a word of the form

$$
a^{-1} C_{1}^{n_{1}} a C_{2}^{n_{2}} a^{-1} C_{1}^{n_{3}} \cdots a^{-1} C_{1}^{n_{r-2}} a C_{2}^{n_{r-1}} a^{-1} C_{1}^{n_{r}}
$$

which does not have to begin with a $C_{2}$-term because $f$ is constant on $C_{1}$. For $h$ the loop in Figure 8 , we here replace $a^{-1} C_{1}^{k} a$ by $h^{k}$ to obtain

$$
h^{n_{1}} C_{2}^{n_{2}} h^{n_{3}} \ldots h^{n_{r-2}} C_{2}^{n_{r-1}} a^{-1} C_{1}^{n_{r}} .
$$

There are no fixed points on $\partial P$ and a single fixed point on $a$ corresponding to the final $a^{-1}$ in the word. The map $f$ must have at least one fixed point because its Lefschetz number is nonzero.

Case $(1,2,2)$. There will be no interior fixed points in this case. The picture for $Y$ is as shown in Figure 9 (see next page). This time we can assume $f(a)=x_{2}$.

Theorem 4.8. If $f:(P, \partial P) \rightarrow(P, \partial P)$ is a boundary inessential map then $M F_{\partial}[f]=N_{\partial}(f)$.

Proof. We use the cw decomposition shown in Figure 4. We can assume that $f(\partial P) \subset\left\{x_{0}, x_{1}, x_{2}\right\}$. First consider the case $\operatorname{Im}_{\partial}(f)=3$. Up to numbering of the components, there are three cases: $(0,1,2)$, $(0,2,1)$ and $(1,2,0)$. In the case $(0,1,2)$, we will show that $f$ is homotopic as a map of pairs to a map which has no fixed points on 


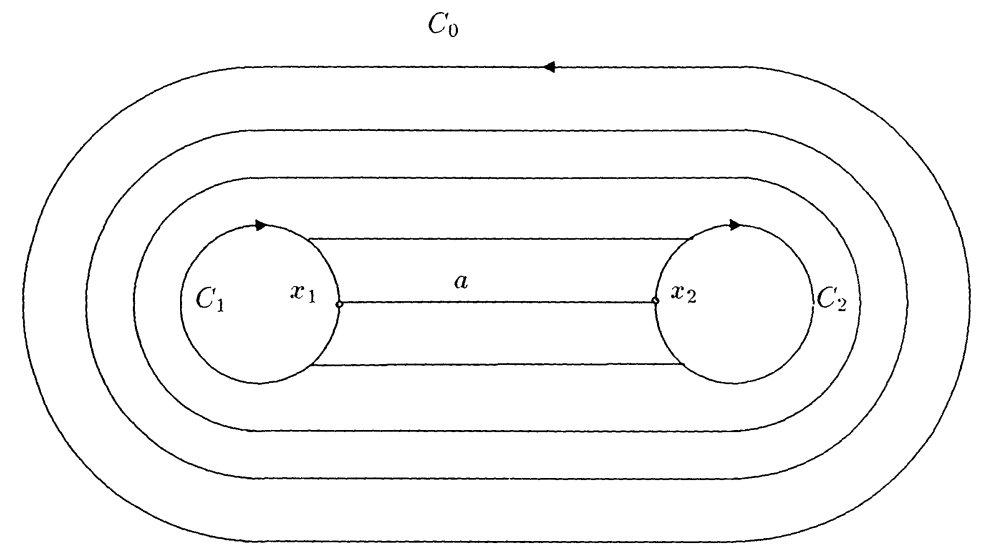

Figure 9

the interior of $P$. Since $\omega_{1} \cup \omega_{2} \cup C_{1} \cup C_{2}$ is a strong deformation retract of $P$ we can assume that $\operatorname{im}(f) \subset\left(\omega_{1} \cup \omega_{2} \cup C_{1} \cup C_{2}\right)$.

We now make $f$ transverse to the cw complex $\omega_{1} \cup \omega_{2} \cup C_{1} \cup C_{2}$. The corresponding picture is shown in Figure 10. Any (innermost) outer circle parallel to $C_{0}$ could be eliminated by a homotopy which replaces it by a pair of circles-one around $C_{1}$ and one around $C_{2}$. The curves $h_{1}, h_{2}$ are chosen to lie in a region outside the circles which maps to $x_{0}$. As in the proof of 4.7 the fixed points of $f$ must lie on $\omega_{1} \cup \omega_{2}$. The effect of $f$ on $\omega_{1}$ is given by a word in $\omega_{1}, \omega_{2}, C_{1}, C_{2}$. Any subword of the form $\omega_{1} C_{1}^{n} \omega_{1}^{-1}$ can be replaced by $h_{1}^{n}$. It is now easy to see that after this replacement the word represents a map which has no fixed point on the interior of $\omega_{1}$. Similarly we may use $h_{2}$ to eliminate the fixed points on $\omega_{2}$. The case $(0,2,1)$ can be treated similarly. For the case $(1,2,0)$ after replacement there will still be an interior fixed point in $\omega_{1}$ coming from the beginning of the word. This fixed point cannot be eliminated, since the Lefschetz number of $f$ is nonzero.

Now suppose that $\operatorname{Im}_{\partial}(f)=2$. Cases $(1,1,2)$ and $(1,2,1)$ can be proved as $(0,1,2)$ and $(1,2,0)$ were above, except that for the $(1,1,2)$ case we need to switch the labels $C_{0}$ and $C_{1}$ in Figure 4 . The fixed point in the $(1,2,1)$ case is again in $\omega_{1}$ and again the Lefschetz number is nonzero. For the $(1,2,2)$ case there will be no fixed points in $\operatorname{int}(P)$-switch $C_{0}$ and $C_{2}$ in Figure 4. Finally suppose $\operatorname{Im}_{\partial}(f)=1$; we can suppose $f$ is of type $(0,0,0)$ and a similar argument demonstrates that $f$ can be homotoped to have no interior fixed points in $P$. 


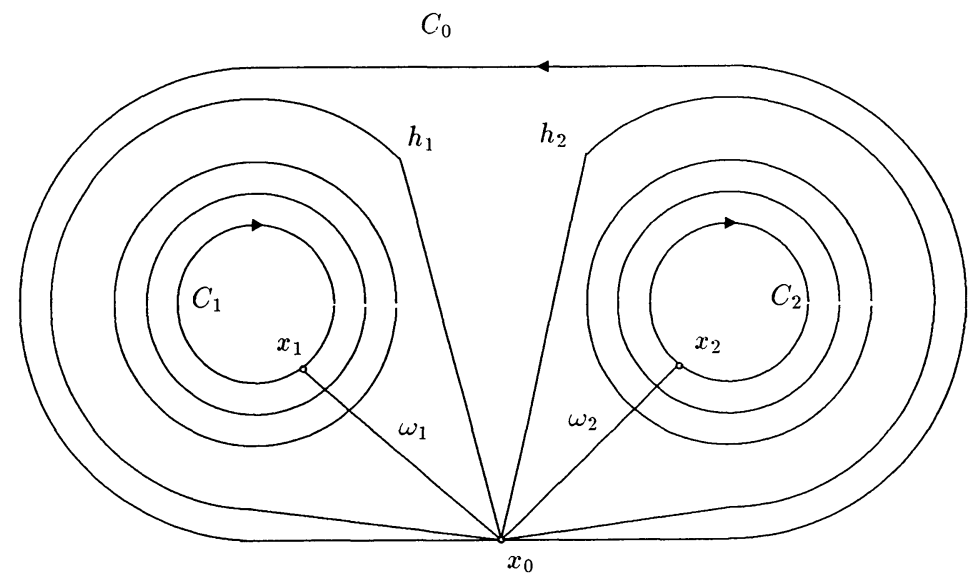

FIGURE 10

THEOREM 4.9. If $f:(P, \partial P) \rightarrow(P, \partial P)$ is a map such that $\operatorname{Im}_{\partial}(f)$ $=3$, then $M F_{\partial}[f]=N_{\partial}(f)$.

Proof. This is immediate from 4.5 and 4.8.

For $f:(P, \partial P) \rightarrow(P, \partial P)$ with $\operatorname{Im}_{\partial}(f)=1$, we will assume that $\partial P$ is mapped to the component $C_{2}$, with $f_{j}: C_{j} \rightarrow C_{2}$ of degree $d_{j}$ with respect to the orientations above. We may in fact assume that $f_{j}=\varphi_{d_{j}}$, as in $\S 2$, and that $f\left(x_{j}\right)=x_{j^{*}}$. Of the three maps $f_{j}$ it cannot happen that one is essential while the others are inessential since after attaching two discs to the domain of $f$ we would get a null homotopy in $P$ of the essential map. For two of the $f_{j}$ essential, it will be sufficient to consider the following cases:

(i) all $f_{j}$ essential, (ii) $f_{0}$ and $f_{2}$ essential, $f_{1}$ inessential, (iii) $f_{0}$ and $f_{1}$ essential, $f_{2}$ inessential.

THEOREM 4.10. In each of cases (i) and (ii) $M F_{\partial}[f]=N_{\partial}(f)$. In case (iii) $M F_{\partial}[f] \leq N_{\partial}(f)+1$.

Proof. We have the arc $a$, the points $b_{j}$ for $j=1,2,3$ and let $Z=C_{1} \cup a \cup C_{2}$ as in Theorem 4.7. We still assume $f$ maps $P$ to $Z$ and is transverse to $Z$, and let $Y=f^{-1}\left(b_{1} \cup b_{2} \cup b_{3}\right)$. We will assume that innermost circles in $Y$ have been removed wherever possible. In each of cases (i) and (ii) we will deform $f$ rel the boundary so that there are no fixed points on $\operatorname{int}(P)$ and we can conclude that $M F_{\partial}[f]=N\left(f_{2}\right)=N(\bar{f})=N_{\partial}(f)$. Furthermore, in the cases where it may be that $d_{2}=1$, we modify $f$ on $C_{2}$ so it has no fixed points there, as in the proof of 4.7 . 


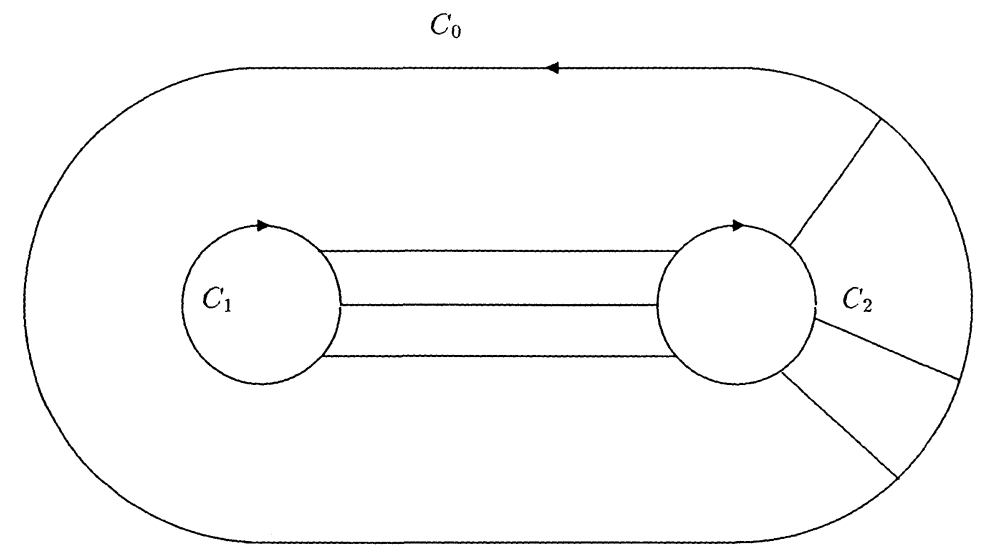

Figure 11

Case (i). After removing innermost circles, $Y$ has the form of Figure 11 with no circles in $Y$, and $f$ now maps all of $P$ to $C_{2}$, so there are certainly no fixed points in $\operatorname{int}(P)$.

Case (ii). The manifold $Y$ is pictured in Figure 8. As in the proof of 4.7 , we represent the image of $a$ by a word. In the present case, it has the form

$$
a^{-1} C_{1}^{n_{1}} a C_{2}^{n_{2}} a^{-1} C_{1}^{n_{3}} a \cdots C_{2}^{n_{r-2}} a^{-1} C_{1}^{n_{r-1}} a C_{2}^{n_{r}} .
$$

We note that the word does not start with a $C_{2}$-term because $f_{1}$ is inessential in this case. Again as in the proof of 4.7, we deform each loop of the form $a^{-1} C_{1}^{k} a$ to $h^{k}$ and obtain the word

$$
h^{n_{1}} C_{2}^{n_{2}} h^{n_{3}} \ldots C_{2}^{n_{r-2}} h^{n_{r-1}} C_{2}^{n_{r}} \text {. }
$$

Thus the only possible fixed point on $a$ is at $x_{2}$.

Case (iii). We see the form of $Y$ in Figure 7. The word describing $f$ on $a$ is this time

$$
C_{2}^{n_{1}} a^{-1} C_{1}^{n_{2}} a C_{2}^{n_{3}} a^{-1} C_{1}^{n_{4}} \cdots a C_{2}^{n_{r-1}} a^{-1} C_{1}^{n_{r}} a
$$

which does not end in a $C_{2}$-term because $f_{2}$ is constant. Similar to the argument in case (ii) of this theorem, we replace terms of the form $a C_{2}^{k} a^{-1}$ by $h^{k}$ to obtain the word

$$
C_{2}^{n_{1}} a^{-1} C_{1}^{n_{2}} h^{n_{3}} C_{1}^{n_{4}} \ldots h^{n_{r-1}} C_{1}^{n_{r}} a .
$$

Recall that the subinterval, at the end of the word, which is mapped to $a$ is made up of three subintervals. The first goes to $x_{1}$, the second to $a$ and the third to $x_{2}$. There is thus a collar region around $C_{2}$ which maps to $x_{2}$. We can change $f$ on the last two subintervals so 
that the $x_{2}$ region disappears and the only fixed point corresponding to $a$ in the word is now $x_{2}$. However, there must be a fixed point $x^{*}$ on the interval of $a$ that is mapped to $a^{-1}$. Since $f_{2}$ is the constant map, we see that every map $f$ of Case (iii) is homotopic as a map of pairs to a map with two fixed points, $x_{2}$ and $x^{*}$. We will show that $x_{2}$ and $x^{*}$ are in the same fixed point class. Let $a_{-}$be the sub-arc of $a$ from $x_{1}$ to $x^{*}$ and $a_{+}$be the sub-arc from $x^{*}$ to $x_{2}$. Let $\omega$ be the path in $Z$ from $x^{*}$ to $x_{2}$ defined as follows:

$$
\omega=a_{-}^{-1} a C_{2}^{k} a^{-1} C_{1}^{n_{2}} a C_{2}^{n_{3}} a^{-1} \cdots a^{-1} C_{1}^{n_{r}} a
$$

where

$$
k=d\left(n_{2}+n_{4}+\cdots+n_{r}\right),
$$

the $n_{j}$ are determined by $f$, as earlier in the proof of this case, and $d$ is the degree of $f_{1}$. Keeping in mind that therefore $f\left(C_{1}^{n_{j}}\right)=C_{2}^{d n_{j}}$ whereas $f\left(C_{2}^{n_{j}}\right)=x_{2}$ and $f\left(a_{-}^{-1}\right)=a_{+} C_{2}^{-n_{1}}$ we have

$$
f(\omega)=a_{+} C_{2}^{-n_{1}} C_{2}^{k} f(a) .
$$

Substituting the first word of this case for $f(a)$, we see that $f(\omega)$ is homotopic to $\omega$ rel the endpoints, so the fixed points are in the same class. Of course $N(\bar{f})=1$ and therefore $N_{\partial}(f)=1$. Since our construction gave a map with two fixed points, we can only claim that $M F_{\partial}[f] \leq 2$ and therefore in this case that $M F_{\partial}[f] \leq N_{\partial}(f)+1$.

To summarize the results of this section

THEOREM 4.11. If $f:(P, \partial P) \rightarrow(P, \partial P)$ is a boundary preserving map of the pants surface, then $M F_{\partial}[f] \leq N_{\partial}(f)+1$ and therefore $P$ is almost $b W$.

5. Conclusion. The obvious question remaining from the preceding section is whether or not the pants surface $P$ is bW. We think not, in fact:

Conjecture 1. The disc with two or more discs removed is not bW.

If our conjecture is correct, then $P$ exhibits a new behavior in Nielsen-Wecken fixed point theory: the difference between the minimum number of fixed points in a homotopy class and the Nielsen number is bounded, but it is not zero. A good candidate for a counterexample to the bW property for $P$ comes from case (iii) of Theorem 4.10. The simplest case corresponds to the word $a^{-1} C_{1} a$. It is 
easy to construct similar examples on a disc with more than two holes.

Conjecture 2. The disc with three or more discs removed is almost bW.

If the disc with three discs removed is almost bW but not $\mathrm{bW}$, it would be interesting to know whether the bound on $M F_{\partial}[f]-N_{\partial}(f)$ is one, as for the pants, or whether the bound must be greater for this, in some sense more complicated, surface. Then one could ask the corresponding question for all these surfaces.

The 2-manifolds with boundary that we have not yet discussed are the Möbius band with one or more discs removed, the torus with one disc removed, the Klein bottle with one or more discs removed and the surfaces obtained by removing discs from the connected sum of three copies of the projective plane. Are these surfaces $b W$, almost $\mathrm{bW}$ or totally non-bW?

\section{REFERENCES}

[BG] R. Brown and R. Greene, An interior fixed point property of the disc, Amer. Math. Monthly, to appear.

[BRS] S. Buoncristiano, C. Rourke and B. Sanderson, A Geometric Approach to Homotopy Theory, London Mathematical Society Lecture Notes Series 18 (Cambridge University Press, 1976).

[J] B. Jiang, Fixed points and braids, II, Math. Ann., 272 (1985), 249-256.

[JG] B. Jiang and J. Guo, Fixed points of surface diffeomorphisms, Pacific J. Math., to appear.

[K1] M. Kelly, Minimizing the number of fixed points for self-maps of compact surfaces, Pacific J. Math., 126 (1987), 81-123.

[K2] Minimizing the cardinality of the fixed point set for self-maps of surfaces with boundary, Michigan Math. J., 39 (1992), 201-217.

[S] H. Schirmer, A relative Nielsen number, Pacific J. Math., 122 (1986), 459473.

[W] F. Wecken, Fixpunktklassen, III, Math. Ann., 118 (1942), 544-577.

Received September 16, 1991 and in revised form December 13, 1991.

UNIVERSITY OF CALIFORNIA

Los ANGELES, CA 90024-1555

E-mail address: rfb@math.ucla.edu

AND

UNIVERSITY OF WARWICK

COVENTRY CV4 7AL, UK

E-mail address: bjs@maths.warwick.ac.uk 


\title{
PACIFIC JOURNAL OF MATHEMATICS \\ Founded by \\ E. F. BECKENBACH (1906-1982) F. WolF (1904-1989)
}

\section{EDITORS}

\section{S. VARADARAJAN \\ (Managing Editor) \\ University of California \\ Los Angeles, CA 90024-1555 \\ vsv@math.ucla.edu}

\section{F. Michael Christ}

University of California

Los Angeles, CA 90024-1555

christ@math.ucla.edu

\section{Herbert Clemens}

University of Utah

Salt Lake City, UT 84112

clemens@math.utah.edu

\author{
THOMAs ENRIGHT \\ University of California, San Diego \\ La Jolla, CA 92093 \\ tenright@ucsd.edu \\ Nicholas ERcolani \\ University of Arizona \\ Tucson, AZ 85721 \\ ercolani@math.arizona.edu \\ R. FINN \\ Stanford University \\ Stanford, CA 94305 \\ finn@gauss.stanford.edu \\ VAUghan F. R. JoNeS \\ University of California \\ Berkeley, CA 94720 \\ vfr@math.berkeley.edu
}

\section{SUPPORTING INSTITUTIONS}

UNIVERSITY OF ARIZONA

UNIVERSITY OF BRITISH COLUMBIA

CALIFORNIA INSTITUTE OF TECHNOLOGY

UNIVERSITY OF CALIFORNIA

UNIVERSITY OF MONTANA

UNIVERSITY OF NEVADA, RENO

NEW MEXICO STATE UNIVERSITY OREGON STATE UNIVERSITY

\author{
UNIVERSITY OF OREGON \\ UNIVERSITY OF SOUTHERN CALIFORNIA \\ STANFORD UNIVERSITY \\ UNIVERSITY OF HAWAII \\ UNIVERSITY OF UTAH \\ WASHINGTON STATE UNIVERSITY \\ UNIVERSITY OF WASHINGTON
}

The Supporting Institutions listed above contribute to the cost of publication of this Journal, but they are not owners or publishers and have no responsibility for its content or policies.

Mathematical papers intended for publication in the Pacific Journal of Mathematics should be in typed form or offset-reproduced (not dittoed), double spaced with large margins. Please do not use built up fractions in the text of the manuscript. However, you may use them in the displayed equations. Underline Greek letters in red, German in green, and script in blue. The first paragraph must be capable of being used separately as a synopsis of the entire paper. In particular it should contain no bibliographic references. Please propose a heading for the odd numbered pages of less than 35 characters. Manuscripts, in triplicate, may be sent to any one of the editors. Please classify according to the 1991 Mathematics Subject Classification scheme which can be found in the December index volumes of Mathematical Reviews. Supply name and address of author to whom proofs should be sent. All other communications should be addressed to the managing editor, or Julie Speckart, University of California, Los Angeles, California 90024-1555.

There are page-charges associated with articles appearing in the Pacific Journal of Mathematics. These charges are expected to be paid by the author's University, Government Agency or Company. If the author or authors do not have access to such Institutional support these charges are waived. Single authors will receive 75 free reprints; joint authors will receive a total of 100 free reprints. Additional copies may be obtained at cost in multiples of 50 .

The Pacific Journal of Mathematics (ISSN 0030-8730) is published monthly except for July and August. Regular subscription rate: $\$ 200.00$ a year (10 issues). Special rate: $\$ 100.00$ a year to individual members of supporting institutions.

Subscriptions, orders for numbers issued in the last three calendar years, and changes of address should be sent to Pacific Journal of Mathematics, P.O. Box 969, Carmel Valley, CA 93924, U.S.A. Old back numbers obtainable from Kraus Periodicals Co., Route 100, Millwood, NY 10546.

The Pacific Journal of Mathematics at P.O. Box 969, Carmel Valley, CA 93924 (ISSN 0030-8730) is published monthly except for July and August. Second-class postage paid at Carmel Valley, California 93924, and additional mailing offices. Postmaster: send address changes to Pacific Journal of Mathematics, P.O. Box 969, Carmel Valley, CA 93924.

PUBLISHED BY PACIFIC JOURNAL OF MATHEMATICS, A NON-PROFIT CORPORATION

This publication was typeset using $\mathcal{A} \mathcal{S} S-\mathrm{T}_{\mathrm{E}} \mathrm{X}$, the American Mathematical Society's $\mathrm{T}_{\mathrm{E}} \mathrm{X}$ macro system.

Copyright (c) 1993 by Pacific Journal of Mathematics 


\section{PACIFIC JOURNAL OF MATHEMATICS}

Volume $158 \quad$ No. $2 \quad$ April 1993

On the extension of Lipschitz functions from boundaries of subvarieties to

201 strongly pseudoconvex domains

K. ADACHI and HiRoshi KAJIMOTO

On a nonlinear equation related to the geometry of the diffeomorphism group 223

DaVid DaI-WAi BaO, JACQUES LAFONTAINE and TUdOR S. RATIU

Fixed points of boundary-preserving maps of surfaces

ROBERT F. BROWN and BRIAN SANDERSON

On orthomorphisms between von Neumann preduals and a problem of Araki 265

L. J. BUNCE and JOHN DAVID MAITLAND WRIGHT

Primitive subalgebras of complex Lie algebras. I. Primitive subalgebras of 273 the classical complex Lie algebras

\section{V. CHEKALOV}

$L^{n}$ solutions of the stationary and nonstationary Navier-Stokes equations in 293 $R^{n}$

\section{ZHI MiN CHEN}

Some applications of Bell's theorem to weakly pseudoconvex domains

XiAO JUN HUANG

On isotropic submanifolds and evolution of quasicaustics

STANISŁAW JANECZKO

Currents, metrics and Moishezon manifolds

SHANYU JI

Stationary surfaces in Minkowski spaces. I. A representation formula

JIANGFAN LI

The dual pair $(U(1), U(1))$ over a $p$-adic field

Courtney Hughes Moen

Any knot complement covers at most one knot complement 